\title{
Measure synchronization in a two-species bosonic Josephson junction
}

\author{
Jing Tian ${ }^{1,2}$, Haibo Qiu ${ }^{1,2,3}$ - Guanfang Wang ${ }^{3}$, Yong $\mathrm{Chen}^{2}$, and Li-bin $\mathrm{Fu}^{2}$, 挝 \\ 1. College of Science, Xi'an University of Posts and Telecommunications, 710121, Xi'an, China \\ 2.Institute of Theoretical Physics, Lanzhou University, 730000, Lanzhou, China and \\ 3.Institute of Applied Physics and Computational Mathematics, 100088, Beijing, China
}

(Dated: August 8, 2018)

\begin{abstract}
Measure synchronization (MS) in a two-species bosonic Josephson junction (BJJ) is studied based on semiclassical theory. Six different scenarios for MS, including two in the Josephson oscillation regime ( 0 phase mode) and four in the self-trapping regime ( $\pi$ phase mode), have been clearly shown. Systematic investigations of the common features behind these different scenarios have been performed. We show that the average energies of the two species merge at the MS transition point. The scaling of the power law near the MS transition has been verified, and the critical exponent is $1 / 2$ for all of the different scenarios for MS. We also illustrate MS in a three-dimensional phase space; from this illustration, more detailed information on the dynamical process can be obtained. Particularly, by analyzing the Poincaré sections with changing interspecies interactions, we find that the two-species BJJ exhibits separatrix crossing behavior at MS transition point, and such behavior depicts the general mechanism behind the different scenarios for the MS transitions. The new critical behavior found in a two-species BJJ is expected to be found in real systems of atomic Bose gases.
\end{abstract}

PACS numbers: $05.45 . \mathrm{Mt}, 64.60 .-\mathrm{i}, 03.75 . \mathrm{Mn}, 45.20 . \mathrm{Jj}$

\section{INTRODUCTION}

Coupled dynamical systems can show a magnificent collective behavior called synchronization [1], a concept first being experimentally shown by Huygens with two marine pendulum clocks in 1665 . In recent decades, many nontrivial features have been revealed [2]. In most such studies, coupled dissipative oscillators are employed whereas research on coupled non-dissipative Hamiltonian systems is still at a primitive stage because of complications originating from Liouville's theorem [3-6]. In the latter system, a new kind of collective phenomenon called measure synchronization (MS) was found. As demonstrated by Hampton and Zanette [7], two coupled Hamiltonian systems experience a dynamical phase transition from a state in which the two Hamiltonian systems visit different phase-space domains to a state in which the two Hamiltonian systems cover an identical phase-space domain as the coupling strength increases. Such phenomena were later investigated in coupled Duffing-, $\varphi_{4^{-}}$, and FrenkelKontorova-type Hamiltonian systems[8-10].

Experimentally, the superconducting Josephson junction (SJJ) is perhaps the most widely studied class in the exploration of synchronization; the superconducting Josephson junction can serve as a prime example of coupled dynamical systems. With recent experimental progress in Bose-Einstein condensates (BECs), a bosonic Josephson junction (BJJ) can be created and controlled by confining single-species BECs in a double well [11]. In a pioneering theoretical study [12], Smerzi et al. mapped a single-species BJJ to a classical pendulum system. Therefore, it is natural to expect that a twospecies BJJ, which consists of a two-species BEC provides a model system to study coupled dynamical systems.

\footnotetext{
*Email: phyqiu@gmail.com
}

†Email: 1bfu@iapcm.ac.cn
The single-species BJJ is of great significance in its own right. The generalized Josephson equations describing the BJJ differ from the ones used for the superconducting Josephson junction by the presence of a nonlinear interaction term [12]. Because of this term, a single-species BJJ can exhibit a counter-intuitive phenomenon called macroscopic quantum self-trapping (MQST). In a detailed analysis of this novel phenomenon [13], the Josephson oscillation (JO) regime and MQST regime can be seen in a phase-plane portrait. Additionally, through an increase in the nonlinear interaction term, the dynamical phase transition from JO to MQST will occur because of the separatrix crossing behavior in the phase space [15-18]. This dynamical phase transition behavior has been studied extensively, both theoretically and experimentally 15 23].

Theoretical analysis has been extended to a two-species BJJ [24-37]. A system of equations for coupled pendula can be derived for the temporal evolution of the relative population and relative phase of each species. Many interesting tunneling effects have been found, including the symmetry restoring phase [27], mixed-Rabi-Josephson oscillation [30], counterflow superfluidity [29], and so on. We have studied collective modes in a two-species BJJ [38]. In addition to phase synchronization, we determined that measure synchronization can also occur. The transitions between different modes can be found by varying the interspecies interaction strength.

In this paper, we perform a systematic investigation on the measure synchronization found in such systems. Six different scenarios for MS are clearly determined. We identified that MS is a continuous phase transition, that the scaling law for the MS transitions was numerically verified, and that the critical exponent is $1 / 2$. Particularly, separatrix crossing has been revealed to be the dynamical mechanism behind the different scenarios for MS by Poincaré section analysis. Because experimental progress has been made in the production of twospecies BECs with tunable intra- and interspecies interactions [39, 40], we expect that a two species BJJ can be realized and 
that the MS can thus be experimentally investigated in the near future.

This paper is organized as follows. A brief description of a two-species BJJ model is given in Section II In Sections 【II. different scenarios of MS are introduced. Section IV] presents a detailed analysis of different MS scenarios. Conclusions are given in Section $\mathbf{V}$

\section{THE MODEL}

A two-species bosonic Josephson junction (BJJ) can be experimentally realized by trapping a binary mixture of BECs in a symmetric double well potential. By assuming the interaction among the atoms is sufficiently weak, with the wellknown two-mode approximation [12-14], the Hamiltonian in the second quantization reads:

$$
\begin{aligned}
\hat{H}= & \frac{u_{a}}{2 N_{a}}\left[\left(\hat{a}_{L}^{\dagger} \hat{a}_{L}\right)^{2}+\left(\hat{a}_{R}^{\dagger} \hat{a}_{R}\right)^{2}\right]+\frac{u_{b}}{2 N_{b}}\left[\left(\hat{b}_{L}^{\dagger} \hat{b}_{L}\right)^{2}+\left(\hat{b}_{R}^{\dagger} \hat{b}_{R}\right)^{2}\right] \\
& -\frac{v_{a}}{2}\left(\hat{a}_{L}^{\dagger} \hat{a}_{R}+\hat{a}_{R}^{\dagger} \hat{a}_{L}\right)-\frac{v_{b}}{2}\left(\hat{b}_{L}^{\dagger} \hat{b}_{R}+\hat{b}_{R}^{\dagger} \hat{b}_{L}\right) \\
& +\frac{u_{a b}}{\sqrt{N_{a} N_{b}}}\left(\hat{a}_{L}^{\dagger} \hat{a}_{L} \hat{b}_{L}^{\dagger} \hat{b}_{L}+\hat{a}_{R}^{\dagger} \hat{a}_{R} \hat{b}_{R}^{\dagger} \hat{b}_{R}\right)
\end{aligned}
$$

where $\hat{a}_{L(R)}^{\dagger}\left(\hat{a}_{L(R)}\right)$ and $\hat{b}_{L(R)}^{\dagger}\left(\hat{b}_{L(R)}\right)$ are the creation (annihilation) operators for the localized modes in the left $(L)$ or right $(R)$ well of different $\operatorname{species}(a$ or $b)$ respectively. $N_{a}$ and $N_{b}$ stand for the particle numbers of species $a$ and b. $u_{\sigma}=\left(4 \pi \hbar \mathrm{a}_{\sigma} N_{\sigma} / m_{\sigma}\right) \int\left|\varphi_{\sigma}\right|^{4} d r, u_{a b}=2 \pi \hbar \mathrm{a}_{a b} \sqrt{N_{a} N_{b}}\left(\frac{1}{m_{a}}+\right.$ $\left.\frac{1}{m_{b}}\right) \int\left|\varphi_{a}\right|^{2}\left|\varphi_{b}\right|^{2} d r$ denote the effective interaction of atomic collision between the same kind of species and between the different species, respectively, with $\sigma=a, b$ as the indication of the species, the interactions can be either repulsive or attractive, depending on the sign of $u$. Both $u_{a}, u_{b}$ and $u_{a b}$ can be tuned by Feshbach technique, as demonstrated by experiments in a mixture of ${ }^{87} \mathrm{Rb}$ and ${ }^{85} \mathrm{Rb}$ [39]. $v_{\sigma}=$ $\int\left[\left(\hbar^{2} / 2 m_{\sigma}\right) \nabla \varphi_{L} \nabla \varphi_{R}+V(r) \varphi_{L} \varphi_{R}\right] d r$ is the effective Rabi frequency describing the coupling between two wells.

Under the semi-classical limit [12-14], dynamics of the system can be described by a classical Hamiltonian $H=\left\langle\Psi_{G P}|\hat{H}| \Psi_{G P}\right\rangle / N$, in which $\left|\Psi_{G P}\right\rangle=\frac{1}{\sqrt{N_{a}}}\left(\alpha_{L} \hat{a}_{L}^{\dagger}+\right.$ $\left.\alpha_{R} \hat{a}_{R}^{\dagger}\right)^{N_{a}}|0,0\rangle \otimes \frac{1}{\sqrt{N_{b}}}\left(\beta_{L} \hat{b}_{L}^{\dagger}+\beta_{R} \hat{b}_{R}^{\dagger}\right)^{N_{b}}|0,0\rangle$ is the collective state of the N-particle system with $N=N_{a}+N_{b}$. Here, $\alpha_{j}=\left|\alpha_{j}\right| e^{i \theta_{a j}}$ and $\beta_{j}=\left|\beta_{j}\right| e^{i \theta_{b j}}(j=L$ or $R)$ are four $c$ numbers which correspond to the probability amplitudes of the two different species of atoms in the two wells. And the conservation of particle numbers of each species requires: $\left|\alpha_{L}\right|^{2}+\left|\alpha_{R}\right|^{2}=1,\left|\beta_{L}\right|^{2}+\left|\beta_{R}\right|^{2}=1$.

By introducing the relative population difference: $S_{a}=$ $\left(\left|\alpha_{L}\right|^{2}-\left|\alpha_{R}\right|^{2}\right), S_{b}=\left(\left|\beta_{L}\right|^{2}-\left|\beta_{R}\right|^{2}\right)$, and the relative phases difference $\theta_{\sigma}=\theta_{\sigma L}-\theta_{\sigma R}$. We obtain the mean-field Hamiltonian [24],

$$
H_{t o t}=H_{a}+H_{b}+H_{I},
$$

it is composed of Hamiltonian $H_{\sigma}(\sigma=a, b)$

$$
H_{\sigma}=\frac{u_{\sigma}}{2} S_{\sigma}^{2}-v_{\sigma} \sqrt{1-S_{\sigma}^{2}} \cos \theta_{\sigma},
$$

and the coupling term

$$
H_{I}=u_{a b} S_{a} S_{b} .
$$

$H_{\sigma}$ is well-known as the mean field Hamiltonian for a single-species BJJ [12, 13]; $H_{I}$ is the coupling term. Thus, a two-species BJJ is similar to two coupled single-species BJJs. It is clear that the coupling occurs because of the presence of the interspecies interaction $u_{a b}$.

The equations of motion can be derived by computing: $\dot{\theta_{\sigma}}=\frac{\partial H}{\partial S_{\sigma}}, \dot{S_{\sigma}}=-\frac{\partial H}{\partial \theta_{\sigma}}$, we obtain:

$$
\begin{gathered}
\dot{\theta_{a}}=u_{a} S_{a}+\frac{v_{a} S_{a}}{\sqrt{1-S_{a}^{2}}} \cos \theta_{a}+u_{a b} S_{b} \\
\dot{S_{a}}=-v_{a} \sqrt{1-S_{a}^{2}} \sin \theta_{a} \\
\dot{\theta_{b}}=u_{b} S_{b}+\frac{v_{b} S_{b}}{\sqrt{1-S_{b}^{2}}} \cos \theta_{b}+u_{a b} S_{a} \\
\dot{S_{b}}=-v_{b} \sqrt{1-S_{b}^{2}} \sin \theta_{b} .
\end{gathered}
$$

The tunneling dynamics of a two-species BJJ can be described with Eqs. (5)-(8). Here, the standard fourth-order Runge-Kutta method is used to obtain a numerical solution. Because we are interested in showing the effects of coupling on the dynamics of each species, the collective motions are presented by projecting the state of the full system onto the individual phase spaces, i.e., we study the trajectories $\left(S_{a}(t), \theta_{a}(t)\right)$ in the phase plane $\left(S_{a}, \theta_{a}\right)$ and the trajectories $\left(S_{b}(t), \theta_{b}(t)\right)$ in the phase plane $\left(S_{b}, \theta_{b}\right)$.

The tunneling dynamics for a single-species BJJ have been extensively studied [12-14, 18- 23], and studies of $H_{\sigma}$ based on the semi-classical theory have shown that there are two distinct dynamic regimes in phase space [12, 13, 23]: the Josephson oscillation regime, and the self-trapping regime with a strong nonlinearity $(u / v>1)$. For simplicity, 0 -phase will be used to stand for the Josephson oscillation, in which $\theta_{\sigma}$ oscillates around $\theta_{\sigma}=0$. And $\pi$-phase stand for the self-trapping, in which $\theta_{\sigma}$ oscillates around $\theta_{\sigma}=\pi$. To show the coupled dynamical behavior of $H_{\sigma}$, we will then categorize the initial configurations of a two-species BJJ into two broad categories:

(i) 0 -phase mode

(ii) $\pi$-phase mode

\section{MS IN 0- AND $\pi$-PHASE MODE}

\section{A. 0-phase mode}

First, we present measure synchronization in the 0-phase mode, that is, the mode in which $\theta_{\sigma}$ oscillates around $\theta_{\sigma}=$ 0 . The initial conditions $\left(S_{a}, \theta_{a}, S_{b}, \theta_{b}\right)$ are $(0.2,0.0,0.4,0.0)$, $u_{a}=u_{b}=1.2$, and $v_{a}=v_{b}=1$. 

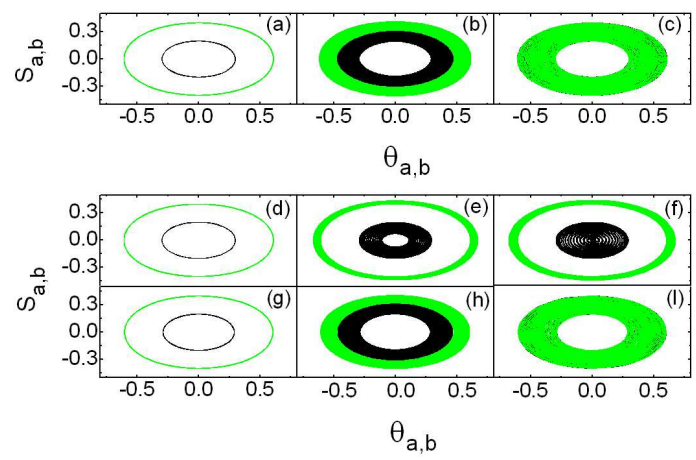

FIG. 1: (color online) Phase-space domains of the two species in the 0 phase mode. The two species are represented by green and black. Initial configuration $\left(S_{a}, S_{b}, \theta_{a}, \theta_{b}\right)$ set to be $(0.2,0.4,0,0)$. (a) $u_{a b}=0$. (b) $u_{a b}=0.0086$. (c) $u_{a b}=0.009$ : MS is achieved. (d) $u_{a b}=0$. (e) $u_{a b}=-0.01$. (f) $u_{a b}=-0.0325$. (g) $u_{a b}=-0.0625$. (h) $u_{a b}=-0.0738$. (i) $u_{a b}=-0.08:$ MS is achieved.

\section{With repulsive interactions $\left(u_{a b}>0\right)$}

Figures 1 $(a)-(c)$ show the evolution process for MS with repulsive interspecies interactions $\left(u_{a b}>0\right)$. By increasing the coupling strength $u_{a b}$, we draw orbits on the $\left(S_{\sigma}, \theta_{\sigma}\right)$, $(\sigma=a, b)$ phase plane of the two subsystems. For $u_{a b}=0$, as shown in Fig. 11a), these initial conditions correspond to two different quasiperiodic orbits, which cover closed curves in green and black. For $u_{a b}>0$, the two closed curves are replaced with two smooth quasi-periodic trajectories wandering in two distinctive phase-space domains, which are ring shaped. As $u_{a b}$ increases, the two phase-space domains first evolve such that the external border of the inner domain approaches the internal border of the outer domain, and the two approach each other until $u_{a b}=0.0086$, at which point the two approaching boundaries are almost in contact [Fig. 11(b)]. Then, a sudden change occurs as $u_{a b}$ increases further, as shown in Fig. 1(c). The two formally well-separated phasespace domains merge and cover the phase-space domains with identical invariant measure [7]. This dynamical phase transition of the two phase-space domains marks the transition to measure synchronization. The evolution process described above is identical to that described previously [7-9], which we will call typical MS hereafter.

\section{With attractive interactions $\left(u_{a b}<0\right)$}

Figures $1(d)-(i)$ show the evolution process with increasing strength of attractive interspecies interactions. As a starting point, in Fig. 11 d), we plot the orbits for each species at zero coupling. For $u_{a b}<0$, as $u_{a b}$ decreases, we see that this evolution process is quite different from the typical MS. The two phase-space domains first evolve in the opposite direction; the internal border of the inner ring approaches the center of the phase-space domain, whereas the external border of the outer ring expands [Fig. 11(e)]. For $u_{a b}=-0.0325$, the internal border of the inner ring finally reaches the center of the phase space [Fig. 11f)]. Then, as $u_{a b}$ decreases, these two rings gradually thinning until $u_{a b}=-0.0625$; at this point, the two rings again become two curves[Fig. 11 (g)], which appear similar to $u_{a b}=0$ [Fig. 11d)]. Additionally, as $u_{a b}$ continues to decrease [Figs. 11 $(g)-(i)$ ], the evolution process becomes identical to that for the typical MS process [as described in Figs. 1 $(a)-(c)$ ].

\section{B. Localized $\pi$-phase mode}

Here, we present measure synchronization in $\pi$-phase mode, in which $\theta_{\sigma}$ oscillates around $\theta_{\sigma}=\pi$. The initial conditions $\left(S_{a}, \theta_{a}, S_{b}, \theta_{b}\right)$ are $(0.2, \pi, 0.4, \pi), u_{a}=u_{b}=1.2$, and $v_{a}=v_{b}=1$.

\section{With repulsive interaction $\left(u_{a b}>0\right)$}

Figures $2(a)-(f)$ show the evolution process for MS with repulsive interspecies interactions $\left(u_{a b}>0\right)$. For $u_{a b}=0$, as shown in Fig. 2]a), these initial conditions correspond to two different quasiperiodic orbits, which cover closed curves in green and black, and the two curves have an inverted-triangle shape. By increasing the coupling strength, we can see that the two inverted triangles broaden in such a way that the two embedded phase-space domains evolve in opposite directions [Fig. 2 (b)], and the internal border of the original inner inverted triangle approaches the center of the phase space. At $u_{a b}=0.0737$, the inner phase-space domain reaches the center [Fig. 2(c)]. Subsequently, the two phase-space domains approach one another until they make contact before the MS transition at $u_{a b}=0.1621$, and in this evolution process, there is also be a moment at which the phase-space domains become closed curves again [Fig. 22 d)]. This scenario is similar to the scenario for attractive interactions in the 0 -phase mode. The most obvious difference is that the phase-space domains no longer have conserved boundaries; as the coupling strength increases, the area expands.

\section{With attractive interaction $\left(u_{a b}<0\right)$}

Figures $2(g)-(i)$ show the scenario with increasing strength of attractive interspecies interactions. This scenario is very similar to the scenario for typical MS. We find that as the coupling strength increases, the two phase-space domains approach each other until MS occurs at $u_{c}=-0.0123$.

\section{Nonlocalized $\pi$-phase mode}

In the $\pi$-phase mode, a new type of coherent evolution process is found, as shown in Fig. 3. For the initial conditions $\left(S_{a}, \theta_{a}, S_{b}, \theta_{b}\right)$ of $(0.2, \pi,-0.4, \pi)$. At $u_{a b}=0$, compared with 

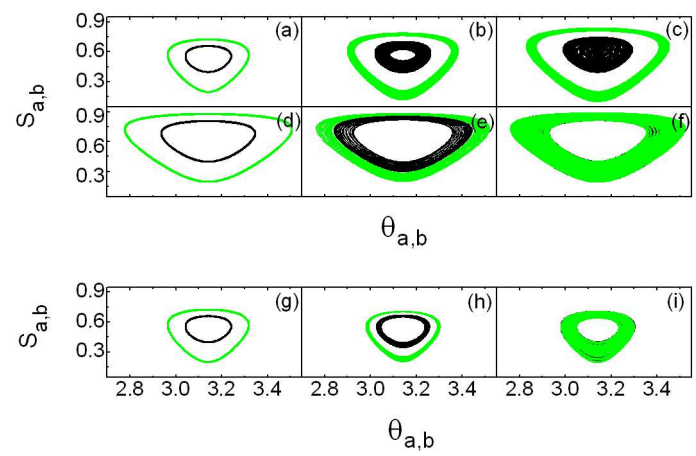

FIG. 2: (color online) Phase-space domains of the two species in the localized $\pi$ phase mode. The two species are represented by green and black. Initial configuration $\left(S_{a}, S_{b}, \theta_{a}, \theta_{b}\right)$ set to be $(0.2,0.4, \pi$, $\pi$ ). (a) $u_{a b}=0$. (b) $u_{a b}=0.03$. (c) $u_{a b}=0.0737$. (d) $u_{a b}=0.1498$. (e) $u_{a b}=0.1621$. (f) $u_{a b}=0.1622$ : MS is achieved for repulsive $u_{a b}$. (g) $u_{a b}=0$. (h) $u_{a b}=-0.01$. (i) $u_{a b}=-0.0123 \mathrm{MS}$ is achieved for attractive $u_{a b}$.

the localized $\pi$-phase mode, these initial conditions also correspond to two closed curves but with one curve on top of the other [Fig. 3(a)].

As the strength of repulsive interspecies interactions increase, the phase-space domains of the two species become more comparable in area until $u_{a b}$ reaches a critical value $\left(u_{a b}=0.0123\right)$ [Fig. 3 $\left.\mathrm{b}\right)$ ]; then, a sudden change occurs, as shown in Fig. 3] (c), and the two phase-space domains have the same area. However, in contrast to Fig. 2(i), the phase-space domain of each species lies symmetrically on both sides of the line $S=0$ [Fig. 3. (c)].

As the strength of attractive interspecies interactions increases, we find another scenario for the transition behavior that ends in a similar state [Fig. 3 ii)]. Interestingly, we note that this scenario has many features in common with the scenario shown in Figs. $2(a)-(g)$. One major difference is the structure of the phase-space domains: one structure goes from top to bottom, whereas the other structure is embedded.

Comparing Fig. 2 and Fig. 3, we can see that each phase diagram corresponds to $u_{a b}$ values with the same magnitudes but with the opposite signs. This result can be understood by analyzing Equation (2)-(4); if we set $s_{a}$ and $s_{b}$ to have opposite signs and let $u_{a b}$ also have a value with opposite sign, the coupling term $H_{I}$ does not change and neither $H_{a}$ or $H_{b}$. The two different initial conditions with opposite signs for the interspecies interactions correspond to the same Hamiltonian and, consequently, have the same dynamic evolution.

\section{ANALYSES}

Below, we will explore the nature of the MS found for a two species BJJ in detail.
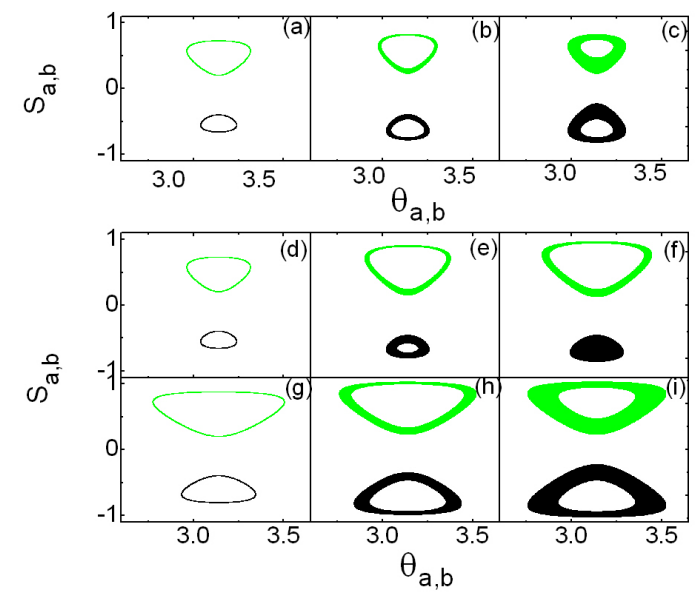

FIG. 3: (color online) Phase-space domains of the two species in the nonlocalized $\pi$ phase mode. The two species are represented by green and black. With the initial conditions $\left(S_{a}, \theta_{a}, S_{b}, \theta_{b}\right)$ taken to be $(0.2, \pi,-0.4, \pi)$ in $\pi$-phase mode. (a) $u_{a b}=0$. (b) $u_{a b}=0.01$. (c) $u_{a b}=0.0123$ : nonlocal MS is achieved for repulsive $u_{a b}$. (d) $u_{a b}=0$. (e) $u_{a b}=-0.03$. (f) $u_{a b}=-0.0737$. (g) $u_{a b}=-0.1498$. (h) $u_{a b}=-0.1621$. (i) $u_{a b}=-0.1622$ : nonlocal MS is achieved for attractive $u_{a b}$.

\section{A. The Energy Characteristics}

For the two groups of MS scenarios that have been found, the 0 - and $\pi$-phase modes, we analysis the energy function for each species: $E_{a, b}$, and observe how the energy function changes with interspecies interactions. Here

$$
\mathrm{E}_{\mathrm{a}, \mathrm{b}}=\frac{u_{a, b}}{2} S_{a, b}^{2}-v_{a, b} \sqrt{1-S_{a, b}^{2}} \cos \theta_{a, b} .
$$

In Fig. 4, we plot the energy function for each species with different interspecies interaction strengths below $u_{c}$ for the initial configuration in the 0 -phase mode and $\pi$-phase mode for repulsive interspecies interaction. Before measure synchronization, $\mathrm{E}_{\mathrm{a}}$ and $\mathrm{E}_{\mathrm{b}}$ do not overlap at all. As $u_{a b}$ continues to increase, the difference between the lower boundary of the initially higher energy species and the upper boundary of the initially lower energy species becomes smaller and smaller. When $u_{a b}$ reaches the transition point $u_{c}, \mathrm{E}_{\mathrm{a}, \mathrm{b}}$ suddenly has the same range of energy variations. This evolution process is shown in Figs. 4( $(a)-(c)$ for the 0-phase mode and in Fig. $4(e)$ $(g)$ for the $\pi$-phase mode. The de-mixing to mixing feature of the MS transitions can be clearly seen in these Figures.

To describe MS in the context of our physical model, the average energy of a single-species BJJ is defined to be:

$$
<\mathrm{E}_{\mathrm{a}, \mathrm{b}}>=\frac{1}{\mathrm{~T}} \int_{0}^{\mathrm{T}} \mathrm{E}_{\mathrm{a}, \mathrm{b}} \mathrm{dt}
$$

In Fig. 5(a), we show the averaged energy $\left\langle E_{a}\right\rangle$ and $<E_{b}>$ as a function of interspecies interactions $u_{a b}$ in the 0 -phase mode. It is clear that there are sharp transitions at $u_{a b}=u_{c}=0.0086$ and $u_{a b}=u_{c}=-0.0738$ for the repulsive and attractive interactions, respectively. Below $u_{c}$ $\left(\left|u_{a b}\right|<\mid u_{c}\right) \mid$, there is a finite difference between $E_{a}$ and $E_{b}$, 

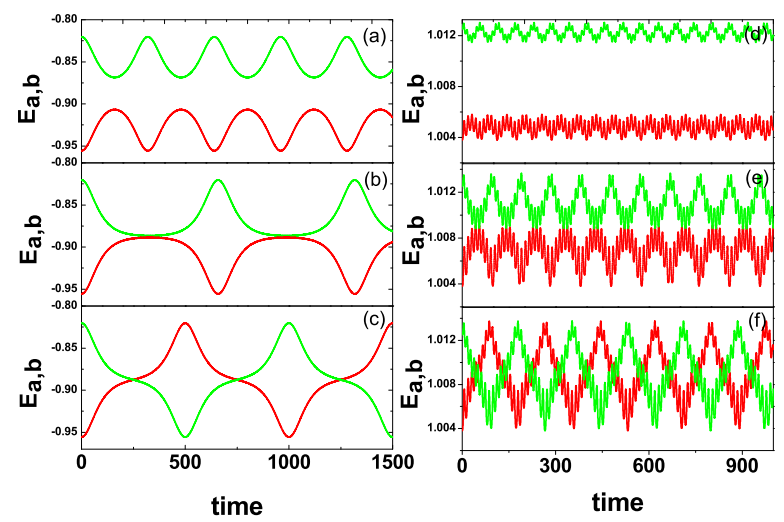

FIG. 4: (color online) Evolution of energy functions for the two species. The left column is for the 0 -phase mode and right column for the $\pi$-phase mode. Before MS is achieved $\left(u_{a b}<u_{c}\right)$, the two species have different energy variations; after MS is achieved $\left(u_{a b} \geq u_{c}\right)$, the two energy variations would be the same. The de-mixing to mixing feature of MS transitions can be clearly seen.
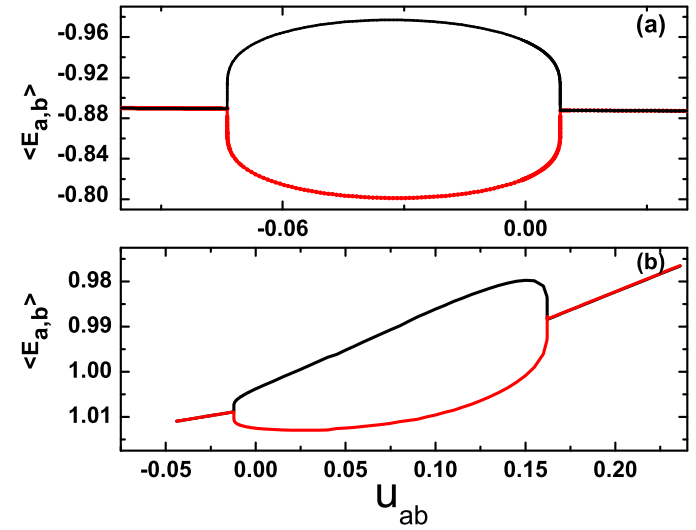

FIG. 5: (color online) The average energies of the two species in the 0 - and $\pi$-phase mode. The two subsystems would have equal averaged energy once MS is achieved. (a). In the 0-phase mode. (b). In the $\pi$-phase mode.

whereas above $u_{c}\left(\left|u_{a b}\right|>\mid u_{c}\right) \mid$, both species have identical average energies. Fig. [5 (b) shows the plot for the $\pi$-phase mode. The correspondence of the MS transition with the sudden merging of the average energies is also clearly shown. In addition, we find that the average energies change in the $\pi$ phase mode, even in the measure-synchronized state, whereas in the 0-phase mode, the average energies remain fixed.

\section{B. The critical behavior}

The critical behavior of MS has been studied previously. In the seminal work [7], Hampton and Zanette introduced an order parameter to study the critical logarithmic singularity; however, they did not find the scaling law and the critical exponent because of the order parameter, which is an aver-
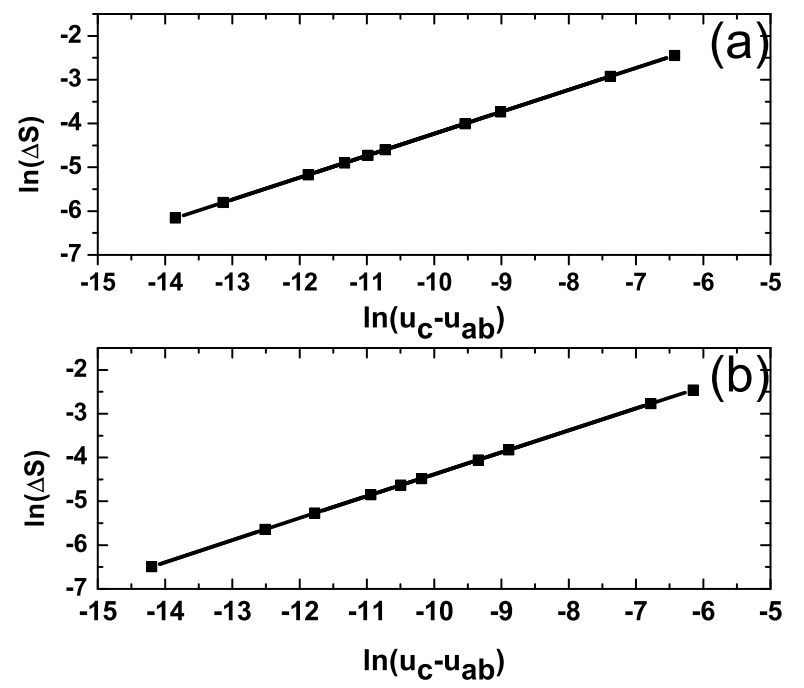

FIG. 6: (color online) Scaling relation of $\triangle S \propto\left(u_{a b}-u_{c}\right)^{\frac{1}{2}}$ for the 0 -phase mode with repulsive interaction (a), and $\pi$-phase mode with repulsive interaction (b).

aged quantity, that was chosen for the calculation. In Ref. [8], through the computation of the interaction energy and the phase dynamics of the oscillators, the scaling law behind MS in coupled $\varphi_{4}$ systems was extensively discussed, and different scaling laws were numerically verified before and after MS. The critical exponents are $1 / 3$ and $1 / 2$.

Here, we studied the critical behavior of MS in a twospecies BJJ. We confirmed that there are scaling laws in this system. As the two phase-space domains approach each other, we noticed that the two phase-domain boundaries are getting close to contact, and there is a scaling relation behind this process. By computing the distance between $(\triangle S)$ the two approaching boundaries on the $S_{a, b}$ axes, we find the scaling relation between $\triangle S$ and $\left(u_{a b}-u_{c}\right)$; this relation is $\triangle S \propto\left(u_{a b}-u_{c}\right)^{\frac{1}{2}}$, and the critical exponent is $1 / 2$. Fig. 6 (a) shows the scaling relation for the 0 phase mode with repulsive interactions and Fig. 6 (b) shows the scaling relation for the $\pi$ phase mode with repulsive interactions. For the other scenarios, we have verified that the critical exponents are all identical and are $1 / 2$.

\section{Three-dimensional description}

Previous work on MS only studied the two-dimensional projected phase-space domains of the coupled-Hamiltonian system. However, this projection could not be a complete description of the dynamical behavior because the dynamics of the two-coupled Hamiltonian actually take place in a four-dimensional phase space $\left(S_{a}, \theta_{a}, S_{b}, \theta_{b}\right)$. In the absence of dissipation, energy constrains the motion of the system to a three-dimensional energy hypersurface of the fourdimensional phase space. To gain the most insight into MS, we can use a three-dimensional description of MS. 
By taking the initial configuration in the 0-phase mode as an example, we provide a three dimensional description of measure synchronization in Fig. 7 First, we choose two different sets of coordinate axes: $\left(S_{\sigma}, \theta_{\sigma}, S_{\bar{\sigma}}\right)$ and $\left(S_{\bar{\sigma}}, \theta_{\bar{\sigma}}, S_{\sigma}\right)$, with $\sigma=a, b$. The corresponding initial conditions and the coupling $u_{a b}$ are identical to those in Fig. 1

With repulsive interspecies interactions $\left(u_{a b}>0\right)$, the evolution process of the three-dimensional phase space is shown in Figs. 7 $(a)-(c)$; these figures show side views of the two manifolds in the three-dimensional phase space representation and top views corresponding to the figures shown in Figs.11a),(b),(c). In Fig.7 (7), for $u_{a b}=0$, there are two wellseparated manifolds, with one manifold around the other. For $u_{a b}=0.0086$, as shown in Fig. 7 (b), the two manifolds are close to each other but are still separated well from one another. However, with $u_{a b}>0.0086$, as shown in Fig. 7 (c) $\left(u_{a b}=0.009\right)$, we see that the two manifolds completely overlap; this overlap indicates the measure-synchronized states in the three-dimensional phase space representation.

With attractive interspecies interactions $\left(u_{a b}<0\right)$, the evolution process of the three-dimensional phase space is shown in Figs. $7(d)-(i)$. The process shown in Figs. $7(d)-(i)$ is not as direct as in case of the repulsive interspecies interactions, because initially the inner phase-space volume shrinks in size (Figs. $7(d)-(g)$ ), then, this volume expands continuously until it achieves the measure-synchronized states (Fig.11i)). There, we can see some behaviors that are not apparent on the twodimensional map; e.g., although Fig. 11 g) and Fig. 11d) appear to be exactly identical, they are actually very different, as shown in the three-dimensional representation: Fig. 7 dd) shows quasi-periodic states, whereas Fig. $7 \mathrm{~g}$ ) shows periodic states. The volume of the synchronized state also apparently changed; however, in the 2-D projection, we cannot see many of these changes.

To summarize, a three-dimensional view of MS is given. It is observed that as MS is attained, the two energy manifolds in the phase space $\left(\left(S_{\sigma}, \theta_{\sigma}, S_{\bar{\sigma}}\right)\right.$ completely overlap. This result provides a more intuitive picture of MS compare with the 2-D projection, and some features that we do not see in the two-dimensional phase space are presented. These features include the difference between the quasiperiodic state and periodic state [Fig. 11(d) and Fig. 11 (g)], and the changing volume of the manifolds as $u_{a b}$ increases can be seen clearly. These results help us understand the measure invariance of the twodimensional phase-space domains after MS is achieved because the phase-space domains can be seen to be the projection of the two energy manifolds on a two-dimensional phase plane.

\section{Poincaré Section Analysis}

Measure synchronization is a dynamical phase transition phenomenon in coupled Hamiltonian systems, so naturally, we ask how this phenomenon occurs? We find that the answer can be revealed through the analysis of the Poincaré maps of the system.

The procedure for our analysis can be demonstrated for the

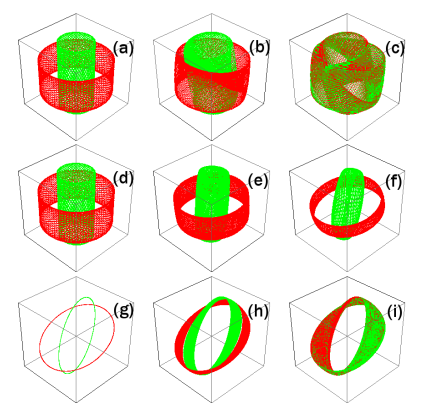

FIG. 7: (Color online) A three-dimensional view of MS for a twospecies BJJ in the 0 phase mode. Two different colors represent two different choices of the three-dimensional axes; the green one is drawn on axes $\left(S_{a}, \theta_{a}, \theta_{b}\right)$ and the red one is drawn on axes $\left(S_{b}, \theta_{b}, \theta_{a}\right)$. (c) and (i) show measure synchronized states.

example of the repulsive interactions in the 0-phase mode [Fig. [ (a)]. First, we solve the canonical equations(5)-(8); then, we take the section slice of $\left(S_{a}, \theta_{a}\right)$ at each time for which $\theta_{b}=0.0$ and $\dot{\theta}_{b}>0$, the section slice taken in this procedure is marked with black dots. Simultaneously, we also take the section slice of $\left(S_{b}, \theta_{b}\right)$ at each time for which $\theta_{a}=0.0$ and $\dot{\theta}_{a}>0$, and this type of section slice is marked with green dots. In Fig. 8 (a), different curves with the same color are drawn for different values of $u_{a b}$ that we chose. For the black dotted curves, with $u_{a b}=0.001$, the corresponding Poincaré section is the innermost, closed, ring-shaped curve. As the coupling intensity increases, this ring-shaped curve expands until $u_{a b}$ reaches $u_{c}\left(u_{c}=0.008621\right)$; at $u_{c}$, the section slice corresponds to the separatrix, which is marked with red dots. For $u_{a b}>u_{c}$, the section slice is shaped like a crescent moon and shrinks in size as $u_{a b}$ increases further. The green dotted curves are drawn for the same chosen set of $u_{a b}$ values; the outermost curve corresponds to the Poincare section for $u_{a b}=0.001$. Conversely, we observe that this ring-shaped Poincaré section shrinks in size before $u_{a b}$ reaches $u_{c}$, and at $u_{c}$, the separatrix is also shown; beyond $u_{c}$, the ring-shaped curve also assumes a crescent moon shape and shrinks in size as $u_{a b}$ increases further. We note that the separatrix marks the transition from the localized to the shared of the phase space. After the measure synchronized of the two coupled Hamiltonian systems for $u_{a b}>u_{c}$, the green-dotted and blackdotted trajectories with the same coupling intensities merge completely.

Similarly, we perform Poincaré section analysis for the other scenarios. Fig. 8 $(\mathrm{b})$ shows the result for the 0-phase mode with attractive interspecies interactions, and Figs. 8(c) and (d) show the results of Poincaré section analysis for the $\pi$-phase mode with attractive interspecies interactions and repulsive interspecies interactions, respectively. We can see that in all cases separatrices mark the onset of measure synchronization.

In summary, through Poincaré section analysis, we have shown that a two-species BJJ exhibits separatrix crossing behavior at the critical interspecies interaction of the MS transition. Therefore, we identified the separatrix crossing to be the 

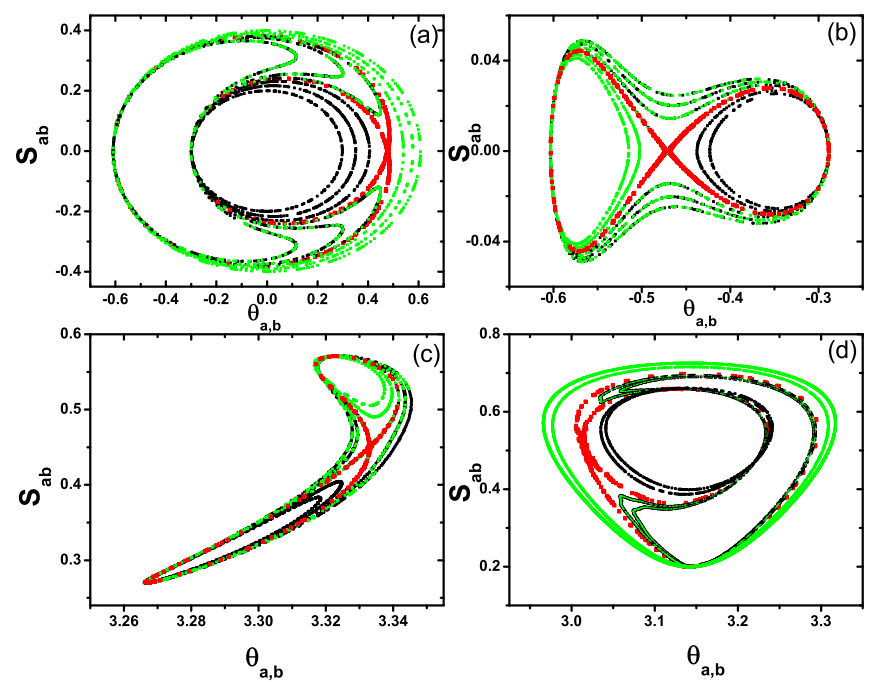

FIG. 8: (color online) Poincaré section analysis. The upper figures and lower figures show the Poincaré section analysis for scenarios in Fig. 1 and in Fig. 2 respectively. The red curves with X-point geometry mark the MS transitions, which correspond to separatrices.

underlying dynamical mechanism of MS.

\section{CONCLUSION}

To summarize, measure synchronization in a two-species BJJ has been systematically studied. Six different scenarios of
MS, including two in the 0 -phase mode, four in the localized and nonlocalized $\pi$-phase mode, have been characterized and some common features behind have been revealed. We have found that the MS transition correspond to the sudden mergence of average energies of the two species. The power law scaling behind the MS transition has been verified, which is the same for the different scenarios, and the critical exponent is $1 / 2$. Furthermore, we have given a three-dimensional view of MS which provides a more intuitive picture of MS. And some features which we will not see in the two dimensional phase space are revealed. In particular, by using the Poincaré section analysis, it has been clearly shown that a two-species BJJ exhibits separatrix crossing behavior at $u_{c}$. We conclude that separatrix crossing is the general mechanism behind the different scenarios of MS transitions found in the two species BJJ.

\section{Acknowledgments}

We thank Qiang Gu for critical reading of the manuscript. This work was supported by the National Natural Science Foundation of China (No.11104217, No.11205121), the Science Plan Foundation office of the Education Department of Shaanxi Province (No.11JK0555), the Youth Foundation of XUPT under Grant No. 0001295, No. 0001287.

\section{References}

[1] Y. Kuramoto, Chemical Oscillations, Waves and Turbulence (Springer, Berlin) (1984); A. Pikovsky, H. Rosenblum and J. Kurths, Synchronization. A Universal Concept in Nonlinear Sciences (Cambridge University Press, Cambridge, England) (2001)

[2] V. Hakim and W. J. Rappel, Phys. Rev. A 46 R7347 (1992); K. Okuda, Physica D (Amsterdam) 63424 (1993); N. Nakagawa and Y. Kuramoto, Prog. Theor. Phys. 89313 (1993); Physica D (Amsterdam) 7574 (1994) ; S. K. Han, C. Kurrer and Y. Kuramoto, Phys. Rev. Lett. 753190 (1995);

[3] H. Morita and K. Kaneko, Phys. Rev. lett. 96050602 (2006).

[4] D. H. Zanette and A. S. Mikhailov, Phys. Lett. A. 235135 (1997).

[5] K. K. Likharev, Dynamics of Josephson Junctions and Circuits Gordon and Breach, New York (1986).

[6] H. A. Cerdeira and S. R. Shenoy, special issue of Physica B 222 (4), 253 (1996).

[7] A. Hampton and D. H. Zanette, Phys. Rev. lett. 832179 (1999).

[8] X. Wang, M. Zhan, C-H. Lai and H. Gang, Phys. Rev. E. 67 066215 (2003).

[9] U. E. Vincent, New J. Phys. 7209 (2005);

[10] J. R. Zhang, H. Jiang, Y. Yang, W. S. Duan and J. M. Chen, Phys. Scr. 86065602 (2012);

[11] M. Albiez, R. Gati, J. Folling, S. Hunsmann , M. Cristiani and M. K. Oberthaler, Phys. Rev. lett. 95010402 (2005).
[12] A. Smerzi, S. Fantoni, S. Giovanazzi and S. R. Shenoy, Phys. Rev. lett. 79, 4950 (1997).

[13] S. Raghavan, A. Smerzi, S. Fantoni, and S. R. Shenoy, Phys. Rev. A. 59, 620 (1999).

[14] A. J. Leggett, Rev. Mod. Phys. 73, 307 (2001).

[15] T. Zibold, E. Nicklas, C. Gross, and M. K. Oberthaler, Phys. Rev. Lett. 105, 204101 (2010).

[16] H. Hennig, D. Witthaut and D. K. Campbell, Phys. Rev. A. 86, R051604 (2012).

[17] G. J. Krahn and D. H. J. O’Dell, J. Phys. B: At. Mol. Opt. Phys. 42205501 (2009).

[18] M. Chuchem, K. Smith-Mannschott, M. Hiller, T Kottos, A. Vardi, and D. Cohen, Phys. Rev. A 82, 053617 (2010).

[19] K. Sakmann, A. I. Streltsov, O. E. Alon, and L. S. Cederbaum, Phys. Rev. Lett. 103, 220601 (2009).

[20] R. Hipolito and A. Polkovnikov, Phys. Rev. A 81, 013621 (2010).

[21] B. Juliá-Díaz, D. Dagnino, M. Lewenstein, J. Martorell, and A. Polls, Phys. Rev. A 81, 023615 (2010).

[22] S. Levy, E. Lahoud, I. Shomroni and J. Steinhauer, Nature (London) 449, 579 (2007); Y. Shin, G. B. Jo, M. Saba, T. A. Pasquini, W. Ketterle and D. E. Pritchard, Phys. Rev. lett. 95, 170402 (2005); L. J. LeBlanc, A. B. Bardon, J. McKeever, M. H. T. Extavour, D. Jervis, J. H. Thywissen, F. Piazza and A. Smerzi, Phys. Rev. Lett. 106, 025302 (2011). 
[23] L-B Fu and J. Liu, Phys. Rev. A. 74063614 (2006).

[24] S. Ashhab and C. Lobo, Phys. Rev. A. 66013609 (2002).

[25] X. Q. Xu, L. H. Lu, Y. Q. Li, Phys. Rev. A 78 , 043609 (2008).

[26] H. T. Ng and P. T. Leung, Phys. Rev. A 71, 013601 (2005).

[27] I. I. Satija, R. Balakrishnan, P. Naudus, J. Heward, M. Edwards, C. W. Clark, Phys. Rev. A 79, 033616 (2009).

[28] G. Mazzarella, M. Moratti, L. Salasnich, M. Salerno, F. Toigo, J. Phys. B: At. Mol. Opt.42, 125301 (2009).

[29] G. Mazzarella, M. Moratti, L. Salasnich, F. Toigo, J. Phys. B: At. Mol. Opt.43, 065303 (2010).

[30] G. Mazzarella, B. Malomed, L. Salasnich, M. Salerno, F. Toigo, J. Phys. B: At. Mol. Opt.44, 035301 (2011).

[31] B. Juliá-Díaz, M. Guilleumas, M. Lewenstein, A. Polls, A. Sanpera, Phys. Rev. A 80, 023616 (2009).

[32] B. Sun, M. S. Pindzola, Phys. Rev. A 80 , 033616 (2009).

[33] A Naddeo, R Citro, J. Phys. B: At. Mol. Opt. Phys 43, 135302 (2010).
[34] M. C. Tichy, J. F. Sherson, K. Mølmer, arXiv: 1204.5896v1.

[35] S. N. Burmistrov, Phys. Rev. A 83, 063627 (2011).

[36] B. Chatterjee, I. Brouzos, L. S. Cao, and P. Schmelcher, Phys. Rev. A 85, 013611 (2012)

[37] L. H. Lu, X. Q. Xu, Y. Q. Li, J. Phys. B: At. Mol. Opt. Phys. 44 145301 (2011).

[38] H. B. Qiu, J. Tian and L-B Fu, Phys. Rev. A. 81043613 (2010).

[39] S. B. Papp and C. E. Wieman, Phys. Rev. Lett. 97, 180404 (2006). S. B. Papp , J. M. Pino and C. E. Wieman, Phys. Rev. Lett. 101, 040402 (2008). G. Thalhammer, G. Barontini, L. De Sarlo, J. Catani, F. Minardi, and M. Inguscio, Phys. Rev. Lett. 100, 210402 (2008). D. J. McCarron, H. W. Cho, D. L. Jenkin, M. P. Köppinger, and S. L. Cornish, Phys. Rev. A 84, 011603(R) (2011).

[40] C. Chin, R. Grimm, P. Julienne, and E. Tiesinga, Rev. Mod. Phys. 82, 1225C1286 (2010). 